\title{
Covariation, Co-Occurrence and Epiphenomenal Correlation of Empirically Based Syndromes in Children and Adolescents
}

\author{
Manfred Döpfner ${ }^{\mathrm{a}} \quad$ Julia Plueck $^{\mathrm{a}}$ Gerd Lehmkuhl $^{\mathrm{a}}$ Michael Huss ${ }^{\mathrm{e}}$ \\ Klaus Lenz $^{\mathrm{b}}$ Ulrike Lehmkuhl $^{\mathrm{b}}$ Fritz Poustka $^{\mathrm{c}}$ Klaus Schmeck ${ }^{f}$ \\ Joerg M. Fegert ${ }^{d}$ The German CBCL Study Group \\ ${ }^{a}$ Department of Child and Adolescent Psychiatry, University of Cologne, Cologne, ${ }^{b}$ Department of Child and \\ Adolescent Psychiatry, Humboldt-University of Berlin, Berlin, 'Department of Child and Adolescent Psychiatry, \\ University of Frankfurt am Main, Frankfurt am Main, ${ }^{d}$ Department of Child and Adolescent Psychiatry, \\ University of Ulm, Ulm, e Department of Child and Adolescent Psychiatry, University of Mainz, Mainz, Germany; \\ fDepartment of Child and Adolescent Psychiatry, University of Basel, Basel, Switzerland
}

\section{Key Words}

Problem behaviour, children, adolescents - Comorbidity ·

Co-occurrence $\cdot$ Child Behaviour Checklist $\cdot$ Epidemiology

\begin{abstract}
Background: This paper determines the co-occurrence and correlations of different problem behaviours in children and adolescents in German clinical and general population samples. Sampling and Methods: The 2 samples were matched by age and gender (each sample $n=1,760$ ). Including both categorical and dimensional models, rates of co-occurrence, relative risks, odds ratios and Pearson correlations were calculated. Results: The bidirectional comorbidity rates ranged from 7.3 to $34.3 \%$ (epidemiological sample) and from 22.5 to $54.8 \%$ (clinical sample). Most correlations between syndrome scales show medium or large effects. Many can be identified as 'epiphenomenal'; partial correlations from each pair, excluding influences of other syndromes, are much lower. Conclusions: This study shows the cross-cultural generalizability of comorbidity rates. The epiphenomenal nature of some comorbidities warrants future attention.
\end{abstract}

\section{KARGER}

Fax +4161306 1234 E-Mail karger@karger.ch www.karger.com (c) 2009 S. Karger AG, Basel

0254-4962/09/0423-0177\$26.00/0

Accessible online at:

www.karger.com/psp

\section{Introduction}

The covariation or co-occurrence of different psychopathological features has been a matter of scientific interest for the last decade [1-5]. 'Comorbidity' is the most commonly used expression that refers to the co-existence of 2 or more distinct disorders in the same individual. It requires distinct definitions of the disorders, as given in the common classification systems DSM-IV and ICD-10. The debate, however, about whether dimensional or categorical approaches should be used to assess deviant behaviour is still a central taxonomic issue. According to the dimensional view, disorders may involve a pattern resulting from quantitative variations on a range of behavioural dimensions [3], rather than qualitative discontinuity between abnormality and normality. Thus, depending on the approach taken, the use of several indices of comorbidity and covariation is probable, including unidirectional and bidirectional comorbidity rates (UCR, $\mathrm{BCR}$ ) and factor indices like relative risks (RR), odds ratios (OR) or Pearson correlations.

Within the categorical model, comorbidity rates as well as prevalence rates depend on the cut-offs for the 
definition of deviant problem behaviour. In 1994, McConaughy and Achenbach [4] published a summary of the findings of 4 epidemiological studies using structured interviews, each using between 126 and 297 subjects. These studies assessed the same diagnostic domains (affective disorders, anxiety disorders, conduct/oppositional disorders and attention deficit disorders), but each of them used different rules in combining information and determining case definitions. In the aggregated sample, McConaughy and Achenbach [4] calculated mean base rates ranging from $4.7 \%$ (affective disorders) to $11.2 \%$ (conduct/oppositional disorders). The mean UCR ranged from 16.0 to $49.7 \%$. The UCR indicates the proportion of subjects who have disorder 'A' given that they exhibit disorder 'B'. Angold et al. [1] showed the wide range of results summarizing the comorbidity rates of 21 general population studies based on structured diagnostic interviews. Due to the confounding effects of different base rates on UCR, McConaughy and Achenbach [4] calculated mean BCR of the reviewed studies in the aggregated samples that ranged from 10.5 to $23.3 \%$. BCR were recommended by them as a symmetrical measure that determines the proportion of subjects who have both disorders ' $\mathrm{A}$ ' and 'B' compared to those subjects who show at least 1 of them.

In studies based on dimensional models with empirically derived syndromes, such as the Child Behaviour Checklist (CBCL) of Achenbach [6], dimensional and categorical indices of co-occurrence can be calculated. Verhulst and van der Ende [5] reported significant Pearson correlations of all pairs of CBCL syndrome scales in their representative Dutch sample of children aged 4-11 years. All correlations are significant and $r$ values range from 0.14 to 0.65 . In this model, the emphasis lies on the covariation of a behaviour range, and not on the covariation of deviant problem behaviour. In addition, Verhulst and van der Ende [5] calculated OR as a categorical measure of co-occurrence using the 90th percentile of each scale to define deviance. The OR ranged from 2.9 to 18.9. This symmetrical factor indicator represents the interaction of 2 variables, specifying the decreased or increased risk for having the second disorder. It is calculated by comparing the odds of having disorder ' $\mathrm{B}$ ' in the group of subjects with disorder ' $\mathrm{A}$ ' with the odds of having disorder ' $\mathrm{B}$ ' in the group without disorder 'A'. In this definition, the technical meaning of 'odds' should not be confused with its informal usage of simply meaning probability. The methodological advantages of calculating OR go along with the disadvantage of it being less concrete. Yang et al. [7] analysed BCR and OR in a representative Taiwanese sample, based on parent ratings using the CBCL. In con- trast to these bidirectional measures, the RR describes by how many times the probability of having disorder ' $\mathrm{B}$ ' increases in subjects affected by disorder ' $\mathrm{A}$ ' compared to those subjects without disorder 'A'. As for UCR, two perspectives can be calculated.

In clinical samples, comorbidity rates are usually higher than in general population samples. This is due to a methodological problem called 'Berkson's bias': the likelihood of referral for subjects with disorders ' $A$ ' and ' $\mathrm{B}$ ' will be a function of the combined likelihood of referral for each disorder separately, irrespective of referral bias. McConaughy and Achenbach [4] tested the effects of 'Berkson's Bias' and reported BCR and OR of the CBCL syndromes classifying deviant behaviour above the 95th percentile. While base rates in the general population sample ranged from 5.6 to $7.7 \%$, in the clinical sample they were from $20.7 \%$ (somatic complaints) to $41.6 \%$ (attention problems). With one exception, the clinical BCR were significantly above those in the general population sample. In both samples, the minimum BCR was found for the co-occurrence of social problems and somatic complaints (10.5 vs. $21.1 \%$ ), and the maximum BCR for aggressive behaviour and delinquent behaviour (30.2 vs. 51.9\%). Despite this bias, clinical samples are still useful for studying comorbidity, especially when dealing with rare syndromes.

Angold et al. [1] pointed out that the high comorbidity rates and correlations found in epidemiological and clinical samples may be caused by epiphenomenal comorbidity: the correlation of 2 syndromes may be explained by the correlation of each of them with 1 or more other syndromes.

The present study was designed to compare different indices of co-occurrence of children's problem behaviour in clinical versus general population samples. Parents' reports of problem behaviour in children and adolescents aged 4-18 years were obtained via the CBCL [6]. These ratings can be scored on empirically derived syndrome scales enabling the calculation of indices for co-occurrence based on dimensional and categorical models of childhood disorders. The study comprises: (1) determination of comorbidity rates in a large nationally representative German general population sample and a matched large sample of referred children from 3 German clinics and a comparison with international findings; (2) effects of 'Berkson's bias' on particular combinations of childhood behaviour problems by comparing different indices of co-occurrence found in those samples; (3) effects of epiphenomenal comorbidity calculating partial correlations between the syndrome pairs. 


\section{Methods}

\section{Instruments}

The CBCL obtains standardized parent reports of 120 items concerning children's problem behaviours rated on 3-point scales ('not true/somewhat true' or 'sometimes true/very true' or 'often true'). The problems are scored in terms of 8 'syndrome scales' described as: withdrawn, somatic complaints, anxious/depressed, social problems, thought problems, attention problems, delinquent behaviour and aggressive behaviour. The first 3 syndromes form the internalizing broadband group, while delinquent behaviour and aggressive behaviour syndromes form the externalizing broadband group. In our samples, parents assessed their child's behaviour using the German version of the CBCL/4-18 [8]. The 8 syndromes were replicated by factor analyses, and their internal consistency coefficients are comparable to those found in the US sample [9]. The mean scale scores in 2 German general population samples were slightly below those in the US sample and below the overall mean scores of 12 cultures [10-12].

\section{Subjects}

The general population sample was drawn from the first nationwide representative PAK-KID-CBCL survey taken in 1994 [12]. Questionnaires were distributed to households using random route methods by trained interviewers, who asked families with children aged 4-18 years to participate in this study. We obtained parents' ratings of 2,856 children aged 4-18 years, with a response rate of $82.2 \%$. Sociodemographic analyses indicated that this sample was representative of parents of 4- to 18-year-old children in Germany (for more details, see [12]). Clinically referred cases, differing from procedure of Achenbach [6], were included in this sample to obtain representative data.

The clinical sample consisted of parent ratings of 3,081 children aged 4-18 years, who were referred to clinics for child and adolescent psychiatry and psychotherapy of the Universities of Berlin (Virchow-Klinikum; 35.7\%), Cologne (26.9\%) and Frankfurt am Main (37.4\%) between 1989 and 1995. The CBCL was part of the standard diagnostic procedure at admission, and subjects were asked to give informed consent. The mean age was $10.2 \pm$ 3.3 years (SD), and $30.1 \%$ of the patients were girls.

Since substantial age and gender differences were found across different cultures, including Germany and the US $[6,10,12]$, each sample was grouped into 4 subsamples split by age (4-10 and 11$18)$ and gender. An equal number of children $(n=440)$ were randomly selected from each subsample; thus, obtaining a matched general population sample (MGPS) of 1,760 children and adolescents and a matched clinical sample (MCS) of equal size. The mean age $\pm S D$ in both groups shows neither a statistically significant difference when set at $\mathrm{p} \leq 0.005$ (MCS $=10.6 \pm 3.3$, MGPS $=10.2 \pm 4.2 ; \mathrm{t}=3.1$, not significant) nor a substantial effect size following Cohen's criteria $(\mathrm{d}=0.11)$ [13].

\section{Analyses}

Calculation of factor indices and comorbidity rates in a categorical approach to CBCL data of dimensional origin is based on cut-offs for classifying cases as deviant. Based on German norms, different cut-offs for syndrome scales ( $\mathrm{T} \geq 67$; $>95$ th percentile) and broadband scales ( $\mathrm{T} \geq 60$; $>85$ th percentile) were applied as proposed by Achenbach [6]. Subjects of MGPS and MCS scoring above the borderline clinical cut-off were determined for each syndrome separately. Two UCR and RR (unidirectional indicators) must be considered for every pair of syndromes because each of the 2 syndromes can be regarded as a basic and as a comorbid syndrome, whereas BCR and OR are bidirectional indicators, and hence only needed to be calculated once for each pair. Differences between comorbidity rates in different samples are tested via 2 $\times 2 \chi^{2}$ tests.

Correlations of scale scores are influenced by the whole variation of scores. Due to the skewed score distribution, the variance is mainly defined by the variation in subclinical ranges. Pearson correlations were computed for each pair of the 8 (raw) syndrome scales. Means of and comparisons between Pearson correlations were computed by $\mathrm{Z}$ transformation [14]. Partial correlations of each pair of syndromes, excluding the influence of the 6 other syndromes, were calculated in order to control for epiphenomenal effects.

\section{Results}

\section{Base Rates, UCR and RR}

In the MGPS, base rates for deviance on each CBCL syndrome ranged from 4.0 to $5.9 \%$ (mean $=5.1 \%$ ), depending on the cut-off being more or less close to the exact 95th percentile (table 1). The UCR ranged from 12.5 to $56.6 \%$. Table 1 also includes means of UCR and RR for both perspectives. For somatic complaints, low mean UCR of 17.9 (as the basic syndrome) and 17.5\% (as the comorbidity) were found. The highest UCR were obtained for attention problems, with other syndromes in both directions (mean UCR $=30.3$ and 33.9\%). Overall, in the MGPS, rather small differences could be discovered between the UCR of both directions (maximum 10.3\%). This may be due to the small differences in base rates of the syndromes, though differences in definitions.

In the MCS (table 1), base rates for deviance ranged from 27.8 to $54.8 \%$ (mean $39.8 \%$ ). The UCR ranged from $31.9 \%$ (social problems with somatic complaints) to $82.4 \%$ (aggressive behaviour with attention problems). There were differences between the 2 directions of the UCR, for example $82.4 \%$ of individuals showing aggressive behaviour also had attention problems, but in reverse not more than $56.0 \%$ of those who had attention problems also showed aggressive behaviour. As the mean UCR indicate, in the MCS differences between the 2 perspectives of the UCR were much stronger compared to the MGPS. This is caused by very different base rates. Moreover, in the MCS, 48 of 56 UCR were significantly higher than in the MGPS ( $\mathrm{p} \leq 0.001$ ). Overall, the mean comorbidity rate in the MCS (57.3\%) was significantly higher than in the MGPS (29.4\%). For broadband scales (externalizing/internalizing), the base rates were $62.2 / 80.3 \%$ within the MCS and 
Table 1. UCR and RR (in parentheses) in the MGPS and the MCS

\begin{tabular}{|c|c|c|c|c|c|c|c|c|c|c|}
\hline \multirow[t]{2}{*}{ Basic syndrome } & \multicolumn{9}{|c|}{ Comorbidity } & \multirow{2}{*}{$\begin{array}{l}\text { Mean UCR } \\
\text { and RR }\end{array}$} \\
\hline & base rates & WD & SC & $\mathrm{AD}$ & SP & $\mathrm{TP}$ & AP & DB & $\mathrm{AB}$ & \\
\hline \multicolumn{11}{|l|}{ Withdrawn (WD) } \\
\hline MGPS & 4.7 & & $15.7(3.5)$ & $56.6(17.6)$ & $38.6(12.7)$ & $24.1(4.8)$ & $34.9(8.5)$ & $19.3(5.9)$ & $33.7(9.1)$ & $31.8(8.9)$ \\
\hline MCS & 31.6 & & $39.9(1.8)^{*}$ & $77.6(2.3)^{*}$ & $59.8(2.0)^{*}$ & $69.8(2.0)^{*}$ & $71.5(1.5)^{*}$ & $41.3(1.8)^{*}$ & $49.0(1.5)$ & $58.4(1.8)$ \\
\hline \multicolumn{11}{|c|}{ Somatic complaints (SC) } \\
\hline MGPS & 5.0 & $14.8(3.5)$ & & $21.6(4.4)$ & $13.6(3.2)$ & $14.8(2.7)$ & $21.6(4.6)$ & $13.6(3.9)$ & $25.0(6.1)$ & $17.9(4.1)$ \\
\hline MCS & 27.8 & $45.3(1.7)^{*}$ & & $67.7(1.7)^{*}$ & $45.5(1.2)^{*}$ & $58.2(1.4)^{*}$ & $62.9(1.2)^{*}$ & $37.1(1.4)^{*}$ & $44.1(1.3)^{*}$ & $51.5(1.4)$ \\
\hline \multicolumn{11}{|c|}{ Anxious/depressed (AD) } \\
\hline MGPS & 5.7 & $46.5(21.4)$ & $18.8(4.5)$ & & $30.7(10.0)$ & $28.7(6.4)$ & $33.7(8.7)$ & $16.8(5.2)$ & $29.7(8.2)$ & $29.3(9.2)$ \\
\hline MCS & 47.6 & $51.6(3.8)$ & $39.5(2.3)^{*}$ & & $55.3(2.2)^{*}$ & $62.8(2.1)^{*}$ & $70.3(1.7)^{*}$ & $37.7(1.8)^{*}$ & $49.8(1.9)^{*}$ & $52.4(2.3)$ \\
\hline \multicolumn{11}{|c|}{ Social problems (SP) } \\
\hline MGPS & 4.7 & $38.6(12.7)$ & $14.5(3.2)$ & $37.3(8.9)$ & & $26.5(5.4)$ & $43.4(11.7)$ & $21.7(6.9)$ & $21.7(5.1)$ & $29.1(7.7)$ \\
\hline MCS & 39.7 & $47.6(2.6)$ & $31.9(1.3)$ & $66.2(1.9)^{*}$ & & $60.8(1.7)^{*}$ & $81.3(2.2)^{*}$ & $44.2(2.3)^{*}$ & $58.8(2.6)^{*}$ & $55.8(2.1)$ \\
\hline \multicolumn{11}{|c|}{ Thought problems (TP) } \\
\hline MGPS & 5.9 & $19.2(5.1)$ & $12.5(2.8)$ & $27.9(6.4)$ & $21.2(5.7)$ & & $27.9(6.7)$ & $22.1(7.6)$ & $17.3(4.0)$ & $21.2(5.5)$ \\
\hline MCS & 44.4 & $48.7(2.8)^{*}$ & $35.7(1.7)^{*}$ & $65.8(2.0)^{*}$ & $53.2(1.9)^{*}$ & & $71.2(1.7)^{*}$ & $40.3(2.0)^{*}$ & $49.2(1.8)^{*}$ & $53.4(2.0)$ \\
\hline \multicolumn{11}{|c|}{ Attention problems (AP) } \\
\hline MGPS & 5.6 & $29.6(9.1)$ & $19.4(4.7)$ & $34.7(8.6)$ & $36.7(13.0)$ & $29.6(6.6)$ & & $26.5(9.8)$ & $35.7(10.8)$ & $30.3(8.9)$ \\
\hline MCS & 54.8 & $41.2(2.1)$ & $31.9(1.4)$ & $61.0(1.9)^{*}$ & $58.9(3.6)^{*}$ & $59.0(2.0)^{*}$ & & $43.4(3.8)$ & $56.0(3.9)^{*}$ & $50.2(2.7)$ \\
\hline \multicolumn{11}{|c|}{ Delinquent behaviour (DB) } \\
\hline MGPS & 4.0 & $22.5(5.7)$ & $16.9(3.8)$ & $23.9(4.8)$ & $25.4(6.6)$ & $32.4(6.8)$ & $36.6(8.6)$ & & $42.3(11.9)$ & $28.6(6.9)$ \\
\hline MCS & 29.0 & $45.0(1.7)^{*}$ & $35.6(1.4)$ & $61.8(1.5)^{*}$ & $60.5(1.9)^{*}$ & $63.0(1.6)^{*}$ & $82.0(1.9)^{*}$ & & $80.8(4.2)^{*}$ & $61.2(2.0)$ \\
\hline \multicolumn{11}{|c|}{ Aggressive behaviour (AB) } \\
\hline MGPS & 5.1 & $31.1(9.4)$ & $24.4(6.2)$ & $33.3(7.8)$ & $20.0(5.1)$ & $20.0(3.9)$ & $38.9(10.3)$ & $33.3(13.6)$ & & $28.7(8.0)$ \\
\hline MCS & 37.2 & $41.7(1.6)$ & $33.0(1.3)$ & $63.7(1.7)^{*}$ & $62.7(2.4)^{*}$ & $60.0(1.6)^{*}$ & $82.4(2.1)^{*}$ & $63.1(7.1)^{*}$ & & $58.1(2.5)$ \\
\hline \multicolumn{11}{|c|}{ Mean UCR and RR } \\
\hline MGPS & 5.1 & $28.9(9.6)$ & $17.5(3.6)$ & $33.6(3.4)$ & $26.6(8.0)$ & $25.2(5.2)$ & $33.9(8.4)$ & $21.9(7.6)$ & $29.5(7.9)$ & \\
\hline MCS & 39.8 & $45.9(2.3)$ & $35.4(1.6)$ & $66.3(1.9)$ & $56.6(2.2)$ & $61.9(1.8)$ & $74.5(1.8)$ & $43.9(2.9)$ & $55.4(2.5)$ & \\
\hline
\end{tabular}

${ }^{*} \mathrm{p} \leq 0.001$.

$16.9 / 18.1 \%$ in the MGPS. Concerning the internalizing and externalizing scales, the UCR for a child described as being deviant were 70.2 and $81.2 \%$ in the MCS and 50.3 and $48.1 \%$ in the MGPS.

Table 1 also contains RR as a second unidirectional indicator of comorbidity. In both samples, all RR differed significantly from 1 . The RR in the MGPS ranged from 2.7 to 21.4 . In contrast to the UCR, nearly all RR were substantially lower in the MCS (overall mean RR $=2.1$ ) than in the MGPS (overall mean RR = 7.1).

\section{$B C R$ and $O R$}

Table 2 shows the BCR and OR for each syndrome paired with every other syndrome obtained for deviant scores in the 2 samples.
While in the MGPS the BCR ranged from 7.3 to $34.3 \%$, in the MCS they varied from 22.2 to $54.8 \%$. As indicated in table 2, in the MGPS all BCR (except 4) were significantly lower than in the MCS ( $\mathrm{p} \leq 0.001)$. The mean BCR showing deviance on any 2 syndromes were $15.9 \%$ in the MGPS and $37.0 \%$ in the MCS. The minimum and maximum mean BCR on any syndrome with 1 specific other syndrome in the MGPS and the MCS were explored for the same syndromes, although on a different level. In both samples the lowest mean BCR were found for somatic complaints (MGPS $=9.7 \%$; MCS $=26.3 \%$ ), while the highest $\mathrm{BCR}$ were found for attention problems (MGPS $=19.1 \%$; MCS $=42.8 \%)$.

On the broadband scales, base rates for deviance were $17.7 \%$ (externalizing) and $16.9 \%$ (internalizing) in the 
Table 2. BCR and OR (in parentheses), and correlations and partial correlations (in parentheses), between syndromes in the MCS (above diagonal) and MGPS (below diagonal)

\begin{tabular}{|c|c|c|c|c|c|c|c|c|}
\hline & WD & SC & $\mathrm{AD}$ & SP & $\mathrm{TP}$ & AP & DB & $\mathrm{AB}$ \\
\hline \multicolumn{9}{|l|}{ Withdrawn (WD) } \\
\hline $\mathrm{BCR}$ and $\mathrm{OR}$ & & $26.9(2.3)^{*}$ & $44.9(6.8)$ & $36.1(3.4)$ & $40.2(4.5)^{*}$ & $35.4(2.8)^{*}$ & $27.4(2.3)^{*}$ & $29.1(2.1)$ \\
\hline $\begin{array}{l}\text { Correlations and } \\
\text { partial correlations }\end{array}$ & & $0.32(0.08)^{+}$ & $0.63(0.44)^{+}$ & $0.41(0.18)^{\#,+}$ & $0.50(0.22)^{\#,+}$ & $0.38(0.04)^{\#}$ & $0.30(0.16)^{\#,+}$ & $0.22(-0.19)^{\#,+}$ \\
\hline \multicolumn{9}{|l|}{ Somatic complaints (SC) } \\
\hline $\mathrm{BCR}$ and $\mathrm{OR}$ & $8.2(4.0)$ & & $33.2(3.1)^{*}$ & $23.1(1.4)^{*}$ & $28.4(2.0)^{*}$ & $26.9(1.6)^{*}$ & $22.2(1.7)^{*}$ & $23.3(1.5)$ \\
\hline $\begin{array}{l}\text { Correlations and } \\
\text { partial correlations }\end{array}$ & $0.34(0.06)$ & & $0.40(0.26)^{+}$ & $0.11(-0.06)^{\#}$ & $0.26(0.06)$ & $0.15(0.00)^{\#}$ & $0.14(0.07)^{\#}$ & $0.09(-0.06)$ \\
\hline \multicolumn{9}{|l|}{ Anxious/depressed (AD) } \\
\hline $\mathrm{BCR}$ and $\mathrm{OR}$ & $34.3(39.2)$ & $11.2(5.3)$ & & $43.1(3.6)^{*}$ & $47.3(4.0)^{*}$ & $48.5(3.4)^{*}$ & $30.6(2.3)^{*}$ & $38.8(2.9)^{*}$ \\
\hline $\begin{array}{l}\text { Correlations and } \\
\text { partial correlations }\end{array}$ & $0.68(0.44)^{+}$ & $0.38(0.13)^{+}$ & & $0.39(0.08)^{+, \#}$ & $0.48(0.18)^{+}$ & $0.40(0.04)^{\#}$ & $0.26(-0.13)^{+, \#}$ & $0.32(0.17)^{+, \#}$ \\
\hline \multicolumn{9}{|l|}{ Social problems (SP) } \\
\hline $\mathrm{BCR}$ and $\mathrm{OR}$ & $23.9(20.0)$ & $7.5(3.6)$ & $20.3(13.7)$ & & $39.6(2.9)^{*}$ & $51.8(7.3)^{*}$ & $34.3(3.4)^{*}$ & $43.6(4.8)^{*}$ \\
\hline $\begin{array}{l}\text { Correlations and } \\
\text { partial correlations }\end{array}$ & $0.54(0.20)^{+}$ & $0.29(0.03)$ & $0.54(0.13)^{+}$ & & $0.34(-0.03)$ & $0.62(0.41)^{+}$ & $0.36(-0.04)$ & $0.47(0.14)^{+}$ \\
\hline \multicolumn{9}{|l|}{ Thought problems (TP) } \\
\hline $\mathrm{BCR}$ and $\mathrm{OR}$ & $12.0(6.0)$ & $7.3(3.0)$ & $16.5(8.5)$ & $13.3(7.0)$ & & $47.6(3.5)^{*}$ & $32.6(2.8)^{*}$ & $37.0(2.6)^{*}$ \\
\hline $\begin{array}{l}\text { Correlations and } \\
\text { partial correlations }\end{array}$ & $0.40(0.08)^{+}$ & $0.23(0.03)$ & $0.45(0.17)^{+}$ & $0.35(0.06)$ & & $0.46(0.21)^{+, \#}$ & $0.36(0.12)^{+}$ & $0.32(-0.03)$ \\
\hline \multicolumn{9}{|l|}{ Attention problems (AP) } \\
\hline $\mathrm{BCR}$ and $\mathrm{OR}$ & $19.1(12.5)$ & $11.4(5.6)$ & $20.6(12.6)$ & $24.8(20.0)$ & $16.8(8.9)$ & & $39.6(5.9)^{*}$ & $50.0(7.5)^{*}$ \\
\hline $\begin{array}{l}\text { Correlations and } \\
\text { partial correlations }\end{array}$ & $0.52(0.04)$ & $0.32(0.05)$ & $0.56(0.11)^{+}$ & $0.62(0.38)^{+}$ & $0.37(0.06)$ & & $0.50(0.05)$ & $0.61(0.31)^{+}$ \\
\hline \multicolumn{9}{|l|}{ Delinquent behaviour (DB) } \\
\hline $\mathrm{BCR}$ and $\mathrm{OR}$ & $11.6(7.0)$ & $8.2(4.3)$ & $11.0(6.0)$ & $13.2(8.5)$ & $15.1(9.5)$ & $18.2(13.0)$ & & $54.8(17.5)^{*}$ \\
\hline $\begin{array}{l}\text { Correlations and } \\
\text { partial correlations }\end{array}$ & $0.43(0.06)$ & $0.29(0.07)$ & $0.45(0.00)$ & $0.41(0.06)$ & $0.36(0.13)^{+}$ & $0.51(0.08)^{+}$ & & $0.72(0.62)^{\#,+}$ \\
\hline \multicolumn{9}{|l|}{ Aggressive behaviour (AB) } \\
\hline BCR and OR & $19.3(13.3)$ & $14.1(7.9)$ & $18.6(11.3)$ & $11.6(6.2)$ & $10.2(4.6)$ & $22.9(16.2)$ & $22.9(19.9)$ & \\
\hline $\begin{array}{l}\text { Correlations and } \\
\text { partial correlations }\end{array}$ & $0.48(0.06)$ & $0.31(0.03)$ & $0.53(0.16)^{+}$ & $0.43(-0.06)$ & $0.33(-0.02)$ & $0.62(0.32)^{+}$ & $0.65(0.44)^{+}$ & \\
\hline
\end{tabular}

The total number of subjects is 1,760 . Partial correlations refer to correlations with the impact of all other syndrome scales from each pair of syndromes excluded.

${ }^{*} \mathrm{p} \leq 0.001$ between BCR in both samples. ${ }^{*} \mathrm{p} \leq 0.001$ between correlations of both samples. ${ }^{+} \mathrm{p} \leq 0.001$, significant partial correlation.

MGPS, and 62.2\% (externalizing) and 71.9\% (internalizing) in the MCS. The BCR between both scales was $60.4 \%$ for the MCS, twice the respective rate for the MGPS (32.6\%).

In the MGPS, all OR were significantly different from 1.0 (table 2). The strongest relation was detected between anxious/depressed and withdrawn. The risk of being described as deviant on both scales was 39.2 times higher than that of being described as deviant on only 1 scale. The OR of 3.0 for thought problems and somatic complaints represented the lowest relation between 2 syn- dromes in this sample. In the MCS, all OR also differed significantly from 1.0, but in contrast they were all lower than in the MGPS. This is similar to the results regarding the RR which varied from 1.4 to 17.5 (MCS mean OR = 3.9 vs. MGPS mean $\mathrm{OR}=10.6$ ). The correlations between BCR and OR were very high in reference to the MGPS $(\mathrm{r}=0.96)$ and also substantial in the MCS $(\mathrm{r}=0.72)$. Despite the differences in the absolute amount between BCR in the clinical and the representative sample, the correlations of the BCR in both samples were also substantial $(r=0.67)$, and the same is true for OR $(r=0.55)$. In the 
MGPS, the mean OR ranged from 4.8 (somatic complaints) to 14.6 (withdrawn), whereas in the MCS the corresponding values were 1.9 (somatic complaints) and 5.6 (aggressive behaviour).

\section{Correlations and Partial Correlations}

In both samples, all scale correlations were significant. In the MGPS and the MCS, 10 and 4 correlations out of the 28 , respectively, were above $r=0.50$; therefore, showing large effects according to Cohen's [13] criteria. In both samples, somatic complaints indicated rather weak correlations with most other syndromes.

In total, 15 of the 28 correlations in the MGPS were significantly higher than in the MCS, whereas the correlation of withdrawn and thought problems in the MGPS was significantly lower than in the MCS (table 2). In the MGPS, the overall mean correlation of $r=0.46(r=0.31$ $0.52)$ did not differ significantly from the one in the MCS of $r=0.44(r=0.22-0.45)$. The correlations of broadband scales externalizing and internalizing were $r=0.59$ (MGPS) and $r=0.31$ (MCS), respectively.

Compared with the simple correlations, in both samples most partial correlations were significantly lower (table 2). Nearly half of the partial correlations remained significant. In both samples, the highest partial correlations were detected for delinquent behaviour and aggressive behaviour (MCS: $r_{p}=0.62$; MPGS: $r_{p}=0.44$ ); aggressive behaviour and attention problems (MCS: $r_{p}=0.31$; MPGS: $r_{p}=0.32$ ); attention problems and social problems (MCS: $r_{p}=0.41 ;$ MPGS: $r_{p}=0.38$ ), and anxious/depressed and withdrawn (MCS/MPGS: $r_{p}=0.44$ ). These relations do not depend on other correlations, whereas the remaining partial correlations explain less than $7 \%$ of common variance. The maximum reduction was found for withdrawn and attention problems $\left(r=0.52\right.$ to $\left.r_{p}=0.04\right)$ in the MGPS, and for attention problems and delinquent behaviour $\left(r=0.50\right.$ to $\left.r_{p}=0.05\right)$ in the MCS. Mean partial correlations for each syndrome with all other syndromes show a similar range in the MGPS $\left(r_{p}=0.06\right.$ to $\left.r_{p}=0.17\right)$ and in the MCS $\left(r_{p}=0.06\right.$ to $\left.r_{p}=0.16\right)$.

\section{Discussion}

The present study provides data regarding co-occurrences of parent-reported problem behaviours of children from a large general population and clinical samples assessed by the same method, as administered in Englishspeaking countries.
The exact base rates for deviance on CBCL syndromes in the MGPS vary from 4.0 to $5.9 \%$. This is closer to the 95th percentile than those reported by McConaughy and Achenbach [4] because we did not exclude clinically referred children from our normative sample. Nevertheless, differences to the US findings and those of Yang et al. [7] from Taiwan additionally can be ignored. Moreover, we also calculated mean base rates from the metaanalysis of epidemiological studies of Angold et al. [1], concerning diagnoses of anxiety disorders (7.4\%), depression (3.1\%), attention deficit disorders (3.2\%) and conduct/oppositional disorders (7.2\%), which are also close to the base rates found in the present study. In the MCS, we explored base rates from 27.8 to $54.8 \%$, whereas McConaughy and Achenbach [4] reported rates from 20.7 to $40.8 \%$.

Comparisons of the present study with the meta-analysis and the empirical study performed by McConaughy and Achenbach [4], as well as the study in the Taiwanese sample [7], based on BCR and using the national cut-offs for defining deviance showed similar ranges in the epidemiological (US: 10.5-30.2\%; Taiwan: 11.0-35.5\%) and also in the clinical samples (US: 21.1-51.9\%). Similar to the findings of McConaughy and Achenbach [4], for most pairs of CBCL syndromes we discovered higher BCR in the MCS than in the MGPS, and somatic complaints indicated the lowest comorbidity rates with other syndromes in both samples. We also noticed the highest comorbidity rate in the MCS for aggressive behaviour with delinquent behaviour (US: 51.9\%). In both samples, high comorbidity rates could be established for the same syndrome pairs as in the US study, especially for aggressive behaviour with attention problems (US: MCS $=47.2 \%$, MGPS $=28.2 \%$ ), attention problems with social problems (US: MCS $=47.8 \%$, MGPS $=26.2 \%$ ) and anxious/ depressed with social problems (US: MCS $=38.5 \%$, MGPS $=23.1 \%$ ).

Furthermore, there is fairly good agreement between our results and the mean BCR calculated by McConaughy and Achenbach [4] for the epidemiological studies using diagnostic categories (CBCL: anxious depressed/delinquent behaviour $=11 \%$ vs. DSM-III: affective/conduct/ oppositional disorders $=16.9 \%$; CBCL: anxious depressed/aggressive behaviour $=18.6 \%$ vs. DSM-III: anxiety disorders with conduct/oppositional disorders = 14.8\%; CBCL: attention problems/delinquent behaviour $=18.2 \%$ vs. DSM-IIII: attention deficit/conduct/oppositional disorders $=23.3 \%$ ). The BCR of $20.6 \%$ for CBCL attention problems/anxiety disorders, however, is nearly twice as high as the comorbidity rates of attention 
deficit disorders with affective disorders (10.5\%) and attention deficit disorders with anxiety disorders (11.8\%).

Comparing the mean UCR that we calculated based on the meta-analysis of epidemiological studies (Angold et al. [1]) to the corresponding UCR in our MGPS demonstrated larger discrepancies. However, the range of the UCR in the single studies of the meta-analyses was very high.

Besides comorbidity rates, the comparisons with other studies employing the CBCL also showed good agreement of the OR. In the MGPS, they vary from 3.0 to 39.2. The highest OR exists between the syndromes anxious/ depressed and withdrawn. Most other OR are 20.0 or lower. McConaughy and Achenbach [4] computed OR from 3.8 to 19.4, Verhulst and van der Ende [5] reported OR between CBCL syndromes in a representative Dutch sample of 2.7-18.9. In the Taiwanese sample [7], OR of 4.1-35.7 were reported. Again, the ranges of the OR and the syndrome pairs with high OR correspond in all 3 studies. However, the agreement with OR based on diagnostic categories is lower. Angold et al. [1] reported median OR for distinct pairs of disorders based on a metaanalysis of epidemiological studies using diagnostic interviews. For nearly all the tested pairs, comparisons of the corresponding pairs of CBCL syndromes with the OR show a significantly higher degree of comorbidity in our MGPS (p $\leq 0.05$ ).

By stating that in clinical samples the much wider range of base rates would influence the magnitude of the OR, McConaughy and Achenbach [4] argue against their calculation. They assume that the OR would be higher in clinical than in general population samples. We calculated OR as well as RR in the MGPS and MCS, and found that both factor indices are reduced in the MCS. This is also an effect of 'Berkson's bias': if individuals come to clinical attention for either of 2 or more disorders they will appear to be correlated in a clinical sample even if they are not in the general population. This increases comorbidity rates and decreases factor indices in clinical samples since there is a high proportion of subjects who have either disorder A or disorder B or both, and there is a low proportion of subjects with no disorder. Differences are especially pronounced by the rate of those individuals having neither disorder $\mathrm{A}$ nor $\mathrm{B}$.

The correlations between the CBCL syndromes as dimensional measures for co-occurrence in the MGPS vary from $r=0.23$ to $r=0.68$. Between the broadband groupings, externalizing and internalizing, the correlation is $\mathrm{r}=0.59$. McConaughy and Achenbach [4] did not compute correlations, but Verhulst and van der Ende [5] did report correlations of CBCL syndrome scales that vary from $r=0.14$ to $r=0.65$ and an externalizing/internalizing correlation of $r=0.47$. As in our study, the highest correlations were found on syndrome pairs reflecting related behaviours, such as aggressive and delinquent behaviour or withdrawn and anxious/depressed, but also on pairs that reflect contrasting behaviours, such as anxious/depressed and aggressive behaviour or attention problems. In a general population sample, Achenbach [6] also reports similar correlations between CBCL syndromes in different age/gender groups.

The highest covariation was found between syndrome pairs within the internalizing groupings (except for somatic complaints) and within the externalizing groupings. However, crossover relations between syndrome scales from the internalizing and the externalizing groupings were also established. When controlling for 'epiphenomenal' factors, however, most of the covariations between the different syndrome pairs seem to be a mere mathematical product of the correlations of both of them with other syndromes. Thus, the correlations of withdrawn with attention problems in the MGPS and attention problems with delinquent behaviour in the MCS decreased from a large effect to none, following Cohen's criteria [13]. These correlations are real, but they are not specific, meaning that in the absence of other comorbidities, children with high scores on one scale are not more likely to have high scores on the second scale compared to children with average scores on the first scale. However, in both samples, medium effects were found for the covariations of delinquent behaviour with aggressive behaviour, aggressive behaviour with attention problems (therefore reproducing results of Yang et al. [7]), attention problems with social problems and anxious/depressed with withdrawn, even after controlling for all other covariations of these syndrome pairs.

To our knowledge, this is the first study concerning this issue (outside the US) that compares 2 large samples. Several coefficients of co-occurrence and comorbidity are calculated, utilizing categorical and dimensional models to compare epidemiological with clinical data and both with international findings. The study clearly replicates results from the US study about co-occurrence and comorbidity of childhood disorders, by applying CBCL syndromes in representative German population and clinical samples. Despite differences in culture, language and health systems in the US and in Germany, the UCR and RR in both clinical samples (with presumably different selection factors) are very similar. The results of the representative sample are also very similar to results 
of the Dutch and the Taiwanese samples. The striking similarities between these studies based on CBCL syndromes demonstrate the cross-cultural generalizability of the comorbidity results, at least in the 4 cultures analysed. By comparison with meta-analyses based on diagnostic categories derived from structured interviews, some similarities in comorbidity indices were found, but also differences were detected. However, the variation of results within these studies using diagnostic categories is also considerable. Altogether, the cross-cultural variation of comorbidity indices in studies using the same instrument (CBCL) in large samples appears to be smaller than the variation of comorbidity rates within one culture (US) when using different assessment instruments.

\section{Limitations}

The analyses are limited to parent ratings of problem behaviour. The study uses CBCL syndromes that are not equivalent to DSM or ICD diagnostic categories, but which are empirically well-established distinct syndromes across different countries and cultures [11, 15]. Parents may have a stronger tendency to be biased than trained clinical interviewers. Therefore, the results may overestimate the covariation of syndromes. In this study, we did not analyse age and gender effects on syndrome covariations, but we controlled for them by matching our samples for both gender and age.

\section{Clinical Implications}

The study replicates the findings that comorbidity in clinical samples is the rule rather than the exception. This is especially true for parents' reports on rating scales. Since structured interviews are less feasible than rating scales as part of a clinical routine, the comorbidity rates based on parents' ratings are of special interest.

The different indices for comorbidity have specific advantages and disadvantages. From a clinical point of view, UCR are of special interest in showing which other comorbid syndromes may be considered if one distinct disorder is present. RR complete this information. Mean UCR and RR supply this information regarding pairs with a more global view, and inform the clinician about the central tendency of a particular syndrome to be comorbid with any of the others. BCR and OR may be more appropriate if comparisons between different disorders with different base rates are needed. Therefore, they may be more relevant for research.

A large amount of the covariation between all the syndrome pairs, however, can be explained by epiphenomenal effects, and not by specific factors of the respective syndrome pairs. The non-epiphenomenal correlations between delinquent behaviour with aggressive behaviour and attention problems with social problems or anxious/ depressed indicate that the reduction of one problem may also have effects on the other problem.

\section{References}

1 Angold A, Costello EJ, Erkanli A: Comorbidity. J Child Psychol Psychiat 1999;40:57-87.

-2 Bird HR, Gould MS, Staghezza BM: Patterns of diagnostic comorbidities in a community sample of children aged 9 through 16 years. J Am Acad Child Adolesc Psychiatry 1993; 32:361-368

3 Caron C, Rutter M: Comorbidity in child psychopathology: concepts, issues and research strategies. J Child Psychol Psychiatry 1991;32:1063-1080.

4 McConaughy SH, Achenbach TM: Comorbidity of empirically based syndromes in matched general population and clinical samples. J Child Psychol Psychiat 1994;35: 1141-1157.

5 Verhulst FC, van der Ende J: 'Comorbidity' in an epidemiological sample: a longitudinal perspective. J Child Psychol Psychiatry 1993; 34:767-783.

6 Achenbach TM: Manual for the Child Behaviour Checklist/4-18 and 1991 Profile. Burlington, University of Vermont, 1991.
7 Yang HJ, Chen WJ, Soong WT: Rates and patterns of comorbidity of adolescent behavioral syndromes as reported by parents and teachers in a Taiwanese nonreferred sample. J Am Acad Child Adolesc Psychiatry 2001; 40:1045-1052.

8 Arbeitsgruppe Deutsche Child Behaviour Checklist: Elternfragebogen über das Verhalten von Kindern und Jugendlichen: deutsche Bearbeitung der Child Behaviour Checklist (CBCL/4-18). 2. Aufl mit deutschen Normen. Köln, Arbeitsgruppe Kinder-, Jugend- und Familiendiagnostik, 1998.

9 Döpfner M, Berner W, Schmeck K, Lehmkuhl G, Poustka F: Internal consistency and validity of the CBCL and the TRF in a German sample - a cross cultural comparison; in Sergeant J (ed): European Approaches to Hyperkinetic Disorder. Zürich, Fotorotar, 1995, pp 51-81.

10 Crijnen AAM, Achenbach TM, Verhulst FC: Comparisons of problems reported by parents of children in 12 cultures: total problems, externalizing, and internalizing. J Am Acad Child Adolesc Psychiatry 1997;36: 1269-1277.
11 Döpfner M, Schmeck K, Poustka F, Berner W, Lehmkuhl G, Verhulst F: Verhaltensauffaelligkeiten von Kindern und Jugendlichen in Deutschland, den Niederlanden und den USA: Eine kulturvergleichende Studie mit der Child Behaviour Checklist. Nervenarzt 1996;67:960-967.

12 Döpfner M, Plueck J, Berner W, Fegert J, Huss M, Lenz K, Schmeck K, Lehmkuhl U, Poustka F, Lehmkuhl G: Psychische Auffaelligkeiten von Kindern und Jugendlichen in Deutschland - Ergebnisse einer repraesentativen Studie: Methodik, Alters-, Geschlechtsund Beurteilereffekte. Z Kinder Jugendpsychiatr 1997;25:218-233.

13 Cohen J: Statistical Power Analysis for the Behavioral Sciences, ed 2. New York, Academic Press, 1988.

14 Glass GV, Stanley JC: Statistical Methods in Education and Psychology. Englewood Cliffs, Prentice Hall, 1970.

15 Verhulst FC, Achenbach TM: Empirically based assessment and taxonomy of psychopathology: cross-cultural applications. A review. Eur Child Adolesc Psychiatry 1995;4: 61-76. 